\title{
AN APPLICATION OF FIXED POINT THEOREMS IN BEST APPROXIMATION THEORY
}

\author{
H.K. PATHAK \\ Department of Mathematics \\ Kalyan Mahavidyalaya \\ Bhilai Nagar (M.P.) 490 006, INDIA \\ Y.J. CHO \\ Department of Mathematics \\ Gyeongsang National University \\ Chinju 660-701, KOREA \\ S.M. KANG \\ Department of Mathematics \\ Gyeongsang National University \\ Chinju 660-701, KOREA
}

(Received February 7, 1996 and in revised form June 18, 1996)

\begin{abstract}
In this paper, we give an application of Jungck's fixed point theorem to best approximation theory, which extends the results of Singh and Sahab et al.
\end{abstract}

KEY WORDS AND PHRASES: Contractive operator, best approximant, compatible mappings, fixed point.

1991 AMS SUBJECT CLASSIFICATION CODES: 54H25, 47H10.

Let $X$ be a normed linear space. A mapping $T: X \rightarrow X$ is said to be contractive on $X$ (resp., on a subset $C$ of $X$ ) if $\|T x-T y\| \leq\|x-y\|$ for all $x, y$ in $X$ (resp., $C$ ). The set of fixed points of $T$ on $X$ is denoted by $F(T)$. If $\bar{x}$ is a point of $X$, then for $0<a \leq 1$, we define the set $D_{a}$ of best $(C, a)$-approximants to $\bar{x}$ consists of the points $y$ in $C$ such that

$$
a\|y-\bar{x}\|=\inf \{\|z-\bar{x}\|: z \in C\} .
$$

Let $D$ denote the set of best $C$-approximants to $\bar{x}$. For $a=1$, our definition reduces to the set $D$ of best $C$-approximants to $\bar{x}$. A subset $C$ of $X$ is said to be starshaped with respect to a point $q \in C$ if, for all $x$ in $C$ and all $\lambda \in[0,1], \lambda x+(1-\lambda) q \in C$. The point $p$ is called the star-centre of $C$. A convex set is starshaped with respect to each of its points, but not conversely. For an example, the set $C=\{0\} \times[0,1] \cup[1,0] \times\{0\}$ is starshaped with respect to $(0,0) \in C$ as the star-centre of $C$, but it is not convex.

In this paper, we give an application of Jungck's fixed point theorem to best approximation theory, which extends the results of Sahab et al. [9] and Singh [10].

By relaxing the linearity of the operator $T$ and the convexity of $D$ in the original statement of Brosowski [1], Singh [10] proved the following:

Theorem 1. Let $C$ be a $T$-invariant subset of a normed linear space $X$. Let $T: C \rightarrow C$ be a contractive operator on $C$ and let $\bar{x} \in F(T)$. If $D \subseteq X$ is nonempty, compact and starshaped, then $D \cap F(T) \neq \emptyset$.

In the subsequent paper [11], Singh observed that only the nonexpansiveness of $T$ on $D^{\prime}=D \cup\{\bar{x}\}$ is necessary. Further, Hicks and Humphries [4] have shown that the assumption $T: C \rightarrow C$ can be weakened to the condition $T: \partial C \rightarrow C$ if $y \in C$, i.e., $y \in D$ is not necessarily in the interior of $C$, where $\partial C$ denotes the boundary of $C$.

Recently, Sahab, Khan and Sessa [9] generalized Theorem 1 as in the following: 
Theorem 2. Let $X$ be a Banach space. Let $T, I: X \rightarrow X$ be operators and $C$ be a subset of $X$ such that $T: \partial C \rightarrow C$ and $\bar{x} \in F(T) \cap F(I)$. Further, suppose that $T$ and $I$ satisfy

$$
\|T x-T y\| \leq\|I x-I y\|
$$

for all $x, y$ in $D^{\prime}, I$ is linear, continuous on $D$ and $I T x=T I x$ for all $x$ in $D$. If $D$ is nonempty, compact and starshaped with respect to a point $q \in F(I)$ and $I(D)=D$, then $D \cap F(T) \cap F(I) \neq \emptyset$.

Recall that two self-maps $I$ and $T$ of a metric space $(X, d)$ with $d(x, y)=\|x-y\|$ for all $x, y \in X$ are said to be compatible on $X$ if

$$
\lim _{n \rightarrow \infty} d\left(I T x_{n}, T I x_{n}\right)\left(=\lim _{n \rightarrow \infty}\left\|I T x_{n}-T I x_{n}\right\|\right)=0
$$

whenever there is a sequence $\left\{x_{n}\right\}$ in $X$ such that $T x_{n}, I x_{n} \rightarrow t$, as $n \rightarrow \infty$, for some $t$ in $X$ ([6]-[8]). We shall use $N$ to denote the set of positive integers and $C l(S)$ to denote the closure of a set $S$.

For our main theorem, we need the following:

Proposition 3. [8] Let $T$ and $I$ be compatible self-maps of a metric space $(X, d)$ with $I$ being continuous. Suppose that there exist real numbers $r>0$ and $a \in(0,1)$ such that for all $x, y \in X$,

$$
d(T x, T y) \leq r d(I x, I y)+a \max \{d(T x, I x), d(T y, I y)\} .
$$

. Then $T w=I w$ for some $w \in X$ if and only if $A=\cap\left\{C l\left(T\left(K_{n}\right)\right): n \in N\right\} \neq \emptyset$, where for each $n \in N$

$$
K_{n}=\left\{x \in X: d(T x, I x) \leq \frac{1}{n}\right\}
$$

On the other hand, using this proposition, Jungck [8] proved the following:

Theorem 4. Let $I$ and $T$ be compatible self-maps of a closed convex subset $C$ of a Banach space $X$. Suppose that $I$ is continuous and linear with $T(C) \subseteq I(C)$. If there exists an $a \in(0,1)$ such that for all $x, y \in C$,

$$
\|T x-T y\| \leq a\|I x-I y\|+(1-a) \max \{\|T x-I x\|,\|T y-I y\|\},
$$

then $I$ and $T$ have a unique common fixed point in $C$.

By using this theorem, we extend Theorem 2 as in the following:

Theorem 5. Let $X$ be a Banach space. Let $T, I: X \rightarrow X$ be operators and $C$ be a subset of $X$ such that $T: \partial C \rightarrow C$ and $\bar{x} \in F(T) \cap F(I)$. Further, suppose that $T$ and $I$ satisfy (2) for all $x, y$ in $D_{a}^{\prime}=D_{a} \cup\{\bar{x}\} \cup E$, where $E=\left\{q \in X: I x_{n}, T x_{n} \rightarrow q,\left\{x_{n}\right\} \subset D_{a}\right\}, 0<a<1, I$ is linear, continuous on $D_{a}$ and $T, I$ are compatible in $D_{a}$. If $D_{a}$ is nonempty, compact and convex, and $I\left(D_{a}\right)=D_{a}$, then $D_{a} \cap F(T) \cap F(I) \neq \emptyset$.

Proof. Let $y \in D_{a}$ and hence $I y$ is in $D_{a}$ since $I\left(D_{a}\right)=D_{a}$. Further, if $y \in \partial C$, then $T y$ is in $C$ since $T(\partial C) \subseteq C$. From (2), it follows that

$$
\begin{aligned}
\|T y-\bar{x}\| & =\|T y-T \bar{x}\| \\
& \leq a\|I y-I \bar{x}\|+(1-a) \max \{\|T y-I y\|,\|T \bar{x}-I \bar{x}\|\} \\
& \leq a\|I y-\bar{x}\|+(1-a)(\|T y-\bar{x}\|+\|I y-\bar{x}\|),
\end{aligned}
$$

which implies $a\|T y-\bar{x}\| \leq\|I y-\bar{x}\|$ and so $T y$ is in $D_{a}$. Thus $T$ maps $D_{a}$ into itself.

By hypothesis, we have $\bar{x}=T \bar{x}=I \bar{x}$. Then Proposition 3 implies that

$$
A=\cap\left\{C l\left(T\left(K_{n}\right)\right): n \in N\right\} \neq \emptyset .
$$

Suppose that $w \in A$. Then for each $n \in N$, there exists $y_{n} \in T\left(K_{n}\right)$ such that $d\left(w, y_{n}\right)<1 / n$. Consequently, for such $n$, we can and do choose $x_{n} \in K_{n}$ such that $d\left(w, T x_{n}\right)<1 / n$ and so $T x_{n} \rightarrow w$. But since $x_{n} \in K_{n}, d\left(T x_{n}, I x_{n}\right)<1 / n$ and therefore $I x_{n} \rightarrow w$. Thus we have

$$
\lim _{n \rightarrow \infty} I x_{n}=\lim _{n \rightarrow \infty} T x_{n}=w .
$$


Therefore, for a sequence $\left\{x_{n}\right\}$ in $D_{a}$ the existence of (3) is guaranteed whenever $D_{a} \subset K_{n}$. Moreover, $w \in E$. Since $I$ and $T$ are compatible and $I$ is continuous, we have $\lim _{n \rightarrow \infty} T I x_{n}=I w$ and $\lim _{n \rightarrow \infty} I^{2} x_{n}=I w$. By (2), we have

$$
\left\|T I x_{n}-\bar{x}\right\|=\left\|T I x_{n}-T \bar{x}\right\| \leq a\left\|I^{2} x_{n}-I \bar{x}\right\|+(1-a) \max \left\{\left\|T I x_{n}-I^{2} x_{n}\right\|,\|T \bar{x}-I \bar{x}\|\right\},
$$

which implies, as $n \rightarrow \infty$,

$$
\|I w-\bar{x}\| \leq a\|I w-\bar{x}\| .
$$

Hence $I w=\bar{x}$. By (2) again, we have

$$
\|T w-\bar{x}\|=\|T w-T \bar{x}\| \leq a\|I w-I \bar{x}\|+(1-a) \max \{\|T w-I w\|,\|T \bar{x}-I \bar{x}\|\},
$$

which gives $\|T w-\bar{x}\| \leq(1-a)\|T w-\bar{x}\|$, and so $T w=\bar{x}$.

Next, we consider

$$
\left\|T w-T x_{n}\right\| \leq a\left\|I w-I x_{n}\right\| \quad+(1-a) \max \left\{\|T w-I w\|,\left\|T x_{n}-I x_{n}\right\|\right\},
$$

which gives $\|\bar{x}-w\| \leq a\|\bar{x}-w\|$ as $n \rightarrow \infty$, and so $\bar{x}=w$, i.e., $w=I w=T w$. By Theorem $4, w$ must be unique. Hence $E=\{w\}$. Then $D_{a}^{*}=D_{a} \cup\{w\}=D_{a}^{\prime}$

Let $\left\{k_{n}\right\}$ be a monotonically non-decreasing sequence of real numbers such that $0 \leq k_{n}<1$ and $\overline{\lim }_{n \rightarrow \infty} k_{n}=1$. Let $\{x$,$\} be a sequence in D_{a}^{\prime}$ satisfying (3). For each $n \in N$, define a mapping $T_{n}: D_{a}^{\prime} \rightarrow D_{a}^{\prime}$ by

$$
T_{n} x_{\jmath}=k_{n} T x_{\jmath}+\left(1-k_{n}\right) p .
$$

It is possible to define such a mapping $T_{n}$ for each $n \in N$ since $D_{a}^{\prime}$ is starshaped with respect to $p \in F(I)$.

Since $I$ is linear, we have

$$
T_{n} I x_{\jmath}=k_{n} T I x_{\jmath}+\left(1-k_{n}\right) p, \quad I T_{n} x_{\jmath}=k_{n} I T x_{\jmath}+\left(1-k_{n}\right) p .
$$

By compatibility of $I$ and $T$, we have for each $n \in N$,

$$
\begin{aligned}
0 & \leq \lim _{j \rightarrow \infty}\left\|T_{n} I x_{j}-I T_{n} x,\right\| \\
& \leq k_{n} \lim _{j \rightarrow \infty}\left\|T I x_{j}-I T x_{\jmath}\right\|+\lim _{j \rightarrow \infty}\left(1-k_{n}\right)\|p-p\| \\
& =0
\end{aligned}
$$

and so

$$
\lim _{j \rightarrow \infty}\left\|T_{n} I x_{j}-I T_{n} x,\right\|=0
$$

whenever $\lim _{3 \rightarrow \infty} I x_{\jmath}=\lim _{\jmath \rightarrow \infty} T_{n} x_{j}=w$ since we have

$$
\begin{aligned}
\lim _{j \rightarrow \infty} T_{n} x_{\jmath} & =k_{n} \lim _{j \rightarrow \infty} T x_{j}+\left(1-k_{n}\right) w \\
& =k_{n} w+\left(1-k_{n}\right) w \\
& =w .
\end{aligned}
$$

Thus, $I$ and $T_{n}$ are compatible on $D_{a}^{\prime}$ for each $n$ and $T_{n}\left(D_{a}^{\prime}\right) \subset D_{a}^{\prime}=I\left(D_{a}^{\prime}\right)$.

On the other hand, by (2), for all $x, y \in D_{a}^{\prime}$, we have, for all $j \geq n$ and $n$ fixed,

$$
\begin{aligned}
\left\|T_{n} x-T_{n} y\right\|= & k_{n}\|T x-T y\| \leq k_{j}\|T x-T y\|<\|T x-T y\| \\
\leq & a\|I x-I y\|+(1-a) \max \{\|T x-I x\|,\|T y-I y\|\} \\
\leq & a\|I x-I y\|+(1-a) \max \left\{\left\|T x-T_{n} x\right\|+\left\|T_{n} x-I x\right\|,\right. \\
& \left.\left\|T y-T_{n} y\right\|+\left\|T_{n} y-I y\right\|\right\} \\
\leq & a\|I x-I y\|+(1-a) \max \left\{\left(1-k_{n}\right)\|T x-p\|+\left\|T_{n} x-I x\right\|,\right. \\
& \left.\left(1-k_{n}\right)\|T y-p\|+\left\|T_{n} y-I y\right\|\right\} .
\end{aligned}
$$


Hence for all $j \geq n$, we have

$$
\begin{aligned}
\left\|T_{n} x-T_{n} y\right\|<a\|I x-I y\|+(1-a) \max \{(1-k)\|T x-p\| \\
\left.+\left\|T_{n} x-I x\right\|,(1-k)\|T y-p\|+\left\|T_{n} y-I y\right\|\right\}
\end{aligned}
$$

Thus, since $\varlimsup_{3 \rightarrow \infty} k_{j}=1$, from (5), for every $n \in N$, we have

$$
\begin{aligned}
\left\|T_{n} x-T_{n} y\right\|= & \varlimsup_{j \rightarrow \infty} a\left\|T_{n} x-T_{n} y\right\| \\
< & \varlimsup_{, \rightarrow \infty}\left[a\|I x-I y\|+(1-a) \max \left\{\left(1-k_{\jmath}\right)\|T x-p\|\right.\right. \\
& \left.\left.+\left\|T_{n} x-I x\right\|,\left(1-k_{\jmath}\right)\|T y-p\|+\left\|T_{n} y-I y\right\|\right\}\right],
\end{aligned}
$$

which implies

$$
\left\|T_{n} x-T_{n} y\right\|=a\|I x-I y\|+(1-a) \max \left\{\left\|T_{n} x-I x\right\|,\left\|T_{n} y-I y\right\|\right\}
$$

for all $x, y \in D_{a}^{\prime}$. Therefore, by Theorem 4 , for every $n \in N, T_{n}$ and $I$ have a unique common fixed point $x_{n}$ in $D_{a}^{\prime}$, i.e., for every $n \in N$, we have

$$
F\left(T_{n}\right) \cap F(I)=\left\{x_{n}\right\}
$$

Now, the compactness of $D_{a}$ ensures that $\left\{x_{n}\right\}$ has a convergent subsequence $\left\{x_{n_{1}}\right\}$ which converges to a point $z$ in $D_{a}$. Since

$$
x_{n_{\mathrm{i}}}=T_{n_{\mathrm{v}}} x_{n_{\mathrm{i}}}=k_{n_{\mathrm{v}}} T x_{n_{\mathrm{i}}}+\left(1-k_{n_{\mathrm{s}}}\right) q
$$

and $T$ is continuous, we have, as $i \rightarrow \infty$ in (6), $z=T z$, i.e., $z \in D_{a} \cap F(T)$.

Further, the continuity of $I$ implies that

$$
I z=I\left(\lim _{i \rightarrow \infty} x_{n_{i}}\right)=\lim _{i \rightarrow \infty} I x_{n_{i}}=\lim _{i \rightarrow \infty} x_{n_{i}}=z,
$$

i.e., $z \in F(I)$. Therefore, we have $z \in D_{a} \cap F(T) \cap F(I)$ and so

$$
D_{a} \cap F(T) \cap F(I) \neq \emptyset \text {. }
$$

This completes the proof.

ACKNOWLEDGEMENT. The first author was supported in part by U.G.C., New Delhi, India, and the second and third authors were supported in part by the Basic Research Institute Program, Ministry of Education, Korea, 1996, Project No. BSRI-96-1405.

\section{REFERENCES}

1. B. Brosowski, Fixpunktsatze in der Approximation-theorie, Mathematica (Cluj) 11 (1969), 195220 .

2. B. Fisher and S. Sessa, On a fixed point theorem of Greguš, Internat. J. Math. \& Math. Sci. 9 (1986), 23-28.

3. M. Greguš, $A$ fixed point theorem in Banach spaces, Boll. Un. Mat. Ital. (5)17-A (1980), 193198.

4. T. L. Hicks and M. D. Humphries, A note on fixed point theorems, J. Approx. Theroy 34 (1982), 221-225.

5. G. Jungck, An iff fixed point criterion, Math. Mag. 49(1) (1976), 32-34.

6. G. Jungck, Compatible mappings and common fixed points, Internat. J. Math. \& Math. Sci. 9 (1986), 771-779.

7. G. Jungck, Common fixed points for commuting and compatible maps on compacta, Proc. Amer. Math. Soc. 103 (1988), 977-983.

8. G. Jungck, On a fixed point theorem of Fisher and Sessa, Internat. J. Math. \& Math. Sci. 13 (1990), 497-500.

9. S. A. Sahab, M. S. Khan and S. Sessa, A result in best approximation theory, J. Approx. Theory 55 (1988), 349-351.

10. S. P. Singh, An application of a fixed point theorem to approximation theory, J. Approx. Theory 25 (1979), 89-90.

11. S. P. Singh, Application of fixed point theorems in approximation theory, "Applied Nonlinear Analysis" (Edited by V. Lakshmikantham), Academic Press, New York, 1979. 


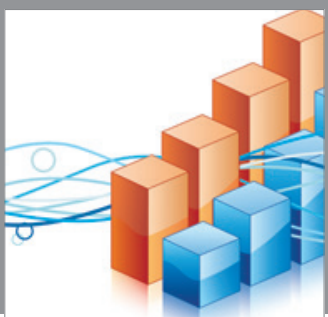

Advances in

Operations Research

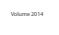

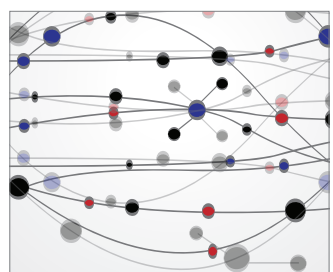

\section{The Scientific} World Journal
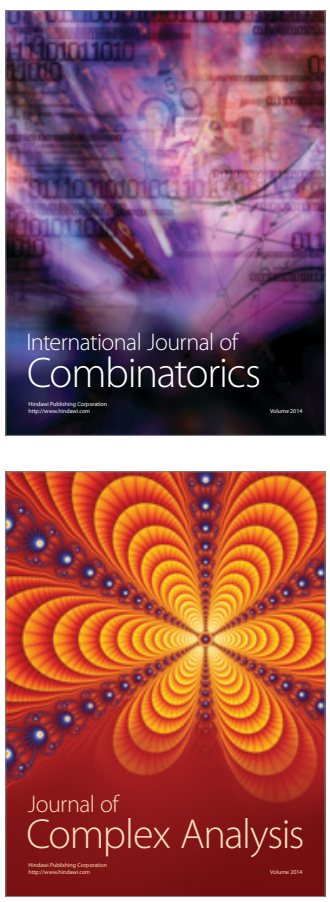

International Journal of

Mathematics and

Mathematical

Sciences
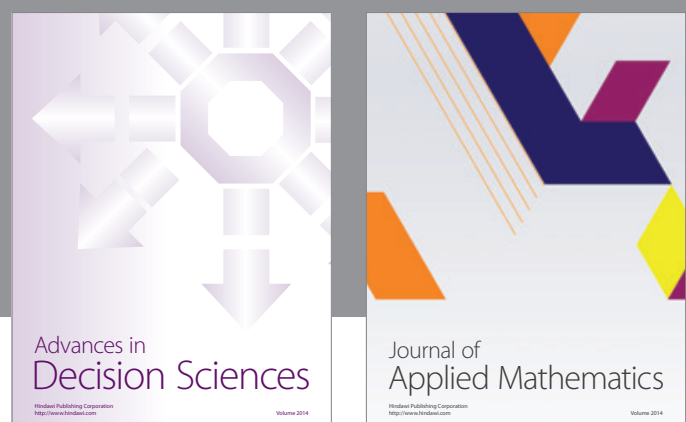

Journal of

Applied Mathematics
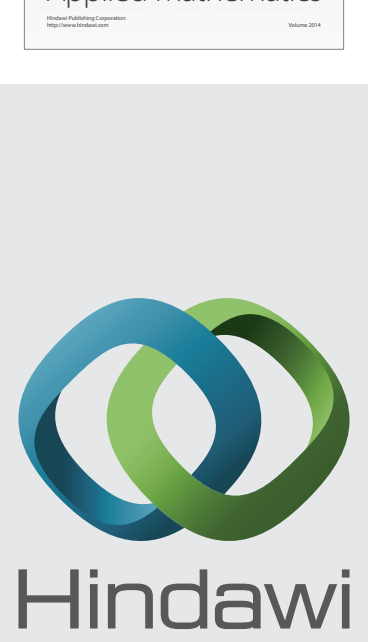

Submit your manuscripts at http://www.hindawi.com
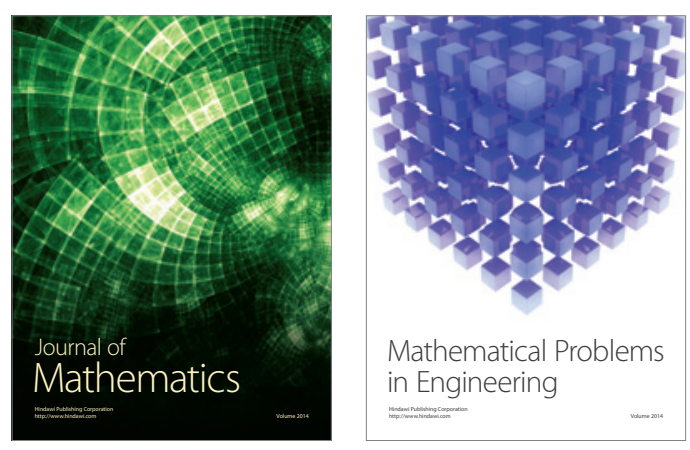

Mathematical Problems in Engineering
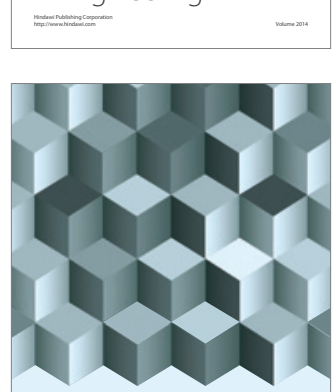

Journal of

Function Spaces
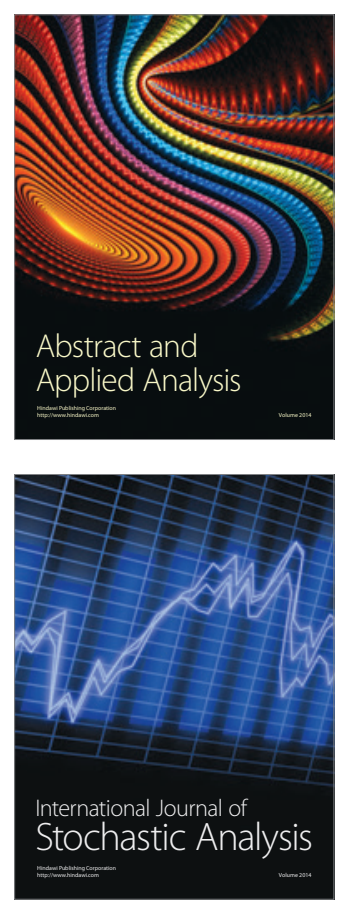

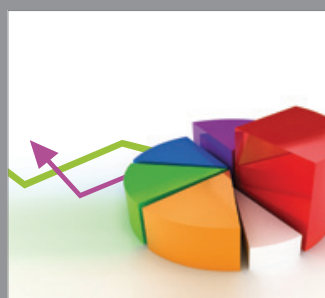

ournal of

Probability and Statistics

Promensencen
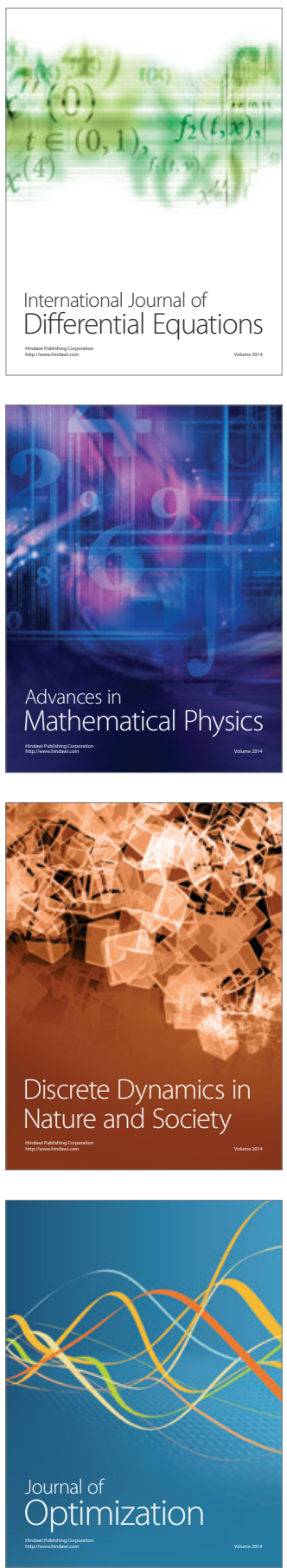